\title{
无过渡金属存在下由邻卤苯酚合成邻卤二芳胺
}

\author{
萑松波 $^{a}$ 何兴瑞 ${ }^{a} \quad$ 孟金鹏 ${ }^{a} \quad$ 顾海宁 ${ }^{a}$ \\ 张培志 $b$ 吴 军*, \\ ( ${ }^{a}$ 浙江大学化学系 杭州 310027) \\ ( ${ }^{b}$ 浙江科技学院生物与化学工程学院 杭州 310012)
}

\begin{abstract}
摘要 报道了一种无过渡金属存在下 $(\mathrm{KOH} / \mathrm{DMSO})$, 以邻卤苯酚和 2-澳- $N$-(芳基)丙酰胺为原料合成邻卤二芳胺的新方 法，其关键步骤涉及到 Smiles 重排反应。该合成方法具有操作简便，原料易得，且在反应中无需使用任何过渡金属试剂 的特点, 具有一定的应用价值. 我们使用该方法以 $23 \% \sim 81 \%$ 的产率合成了一系列邻卤二芳胺.

关键词 无过渡金属反应; 邻卤苯酚; 邻卤二芳胺; Smiles 重排反应
\end{abstract}

\section{Transition-Metal-Free Synthesis of $o$-Halodiarylamines from o-Halophenols}

\author{
Lin, Songbo ${ }^{a}$ \\ He, Xingrui ${ }^{a}$ \\ Meng, Jinpeng ${ }^{a}$ \\ $\mathrm{Gu}$, Haining ${ }^{a}$ \\ Zhang, Peizhi ${ }^{b}$ \\ $\mathrm{Wu}, \mathrm{Jun}^{*, a}$ \\ $\left({ }^{a}\right.$ Department of Chemistry, Zhejiang University, Hangzhou 310027) \\ ( ${ }^{b}$ School of Biological and Chemical Engineering, Zhejiang University of Science and Technology, Hangzhou 310012)
}

\begin{abstract}
A metal-free method (KOH/DMSO) for the synthesis of $o$-halodiarylamines from $o$-halophenols and 2-bromo- $N$ arylpropanamide via Smiles rearrangement reaction as a key step has been developed in this paper. This method has advantages of simple and efficient operation, easy available starting materials and transition-metal-free conditions. It exhibits the certain application value. Using this method, a series of $o$-halodiarylamines have been synthesized in $23 \% \sim 81 \%$ yields.
\end{abstract}

Keywords transition-metal-free reaction; $o$-halophenols; $o$-halodiarylamines; Smiles rearrangement reaction

由于邻卤二芳胺可以作为许多天然产物、功能分子 和药物的关键前体化合物, 因此邻卤二芳胺的合成受到 了有机化学家的关注 ${ }^{[1]}$. 如 Scheme 1 所示, 二芳胺的合 成主要有两个途径: 一是钯催化的卤代苯与苯胺的偶联 反应(Buchwald-Hartwig reaction) ${ }^{[2]}$; 另一种是铜催化的 苯硼酸与苯胺的偶联反应(Chan-Lam coupling $)^{[3]}$. 然而 用上述方法在合成邻卤二芳胺的时候遇到了一些困难, 因为碳-甾键在过渡金属催化的条件下较敏感，易发生 副反应. 此外, 过渡金属通常比较昂贵, 并且对人体和 环境有一定的负面影响 ${ }^{[4]}$. 因此, 通过非过渡金属的方 法实现邻卤二芳胺的合成就变得尤为迫切.
近些年，非过渡金属的方法实现邻卤二芳胺的研究 取得了显著的进展. 2003 年, Larock 小组 ${ }^{[5]}$ 报道了从芳 基甲磺酸酯出发，通过苯炔中间体建立了一种非过渡金 属催化 $N$-芳基类化合物合成邻卤二芳胺的方法. 2007 年, Wood 小组 ${ }^{6}{ }^{6}$ 报道了用二芳基碘鎓盐构建二芳胺的方 法. 2012 年 Rodrigues 等 ${ }^{[7]}$ 用碘使 2-环已基酮胺基化和芳 构化来构建邻碘二芳胺. 然而, 这些非过渡金属的方法 都需要相对昂贵的原料, 并且芳环的多样性也很有限. 因此，高效、通用、易操作地合成邻卤二芳胺类化合物 依然存在着挑战. 本文报道了一种从邻卤苯酚出发, 在 非过渡金属存在下合成邻卤二芳胺类化合物的新方法.

\footnotetext{
* Corresponding author. E-mail: wujunwu@zju.edu.cn Received January 5, 2017; revised February 25, 2017; published online March 1, 2017. China (No. 31471807).

公益性行业(农业)科研专项(No. 201403030)、国家自然科学基金(No. 31471807)资助项目.
}

Project supported by the Special Fund for Agro-scientific Research in the Public Interest (No. 201403030) and the National Natural Science Foundation of 


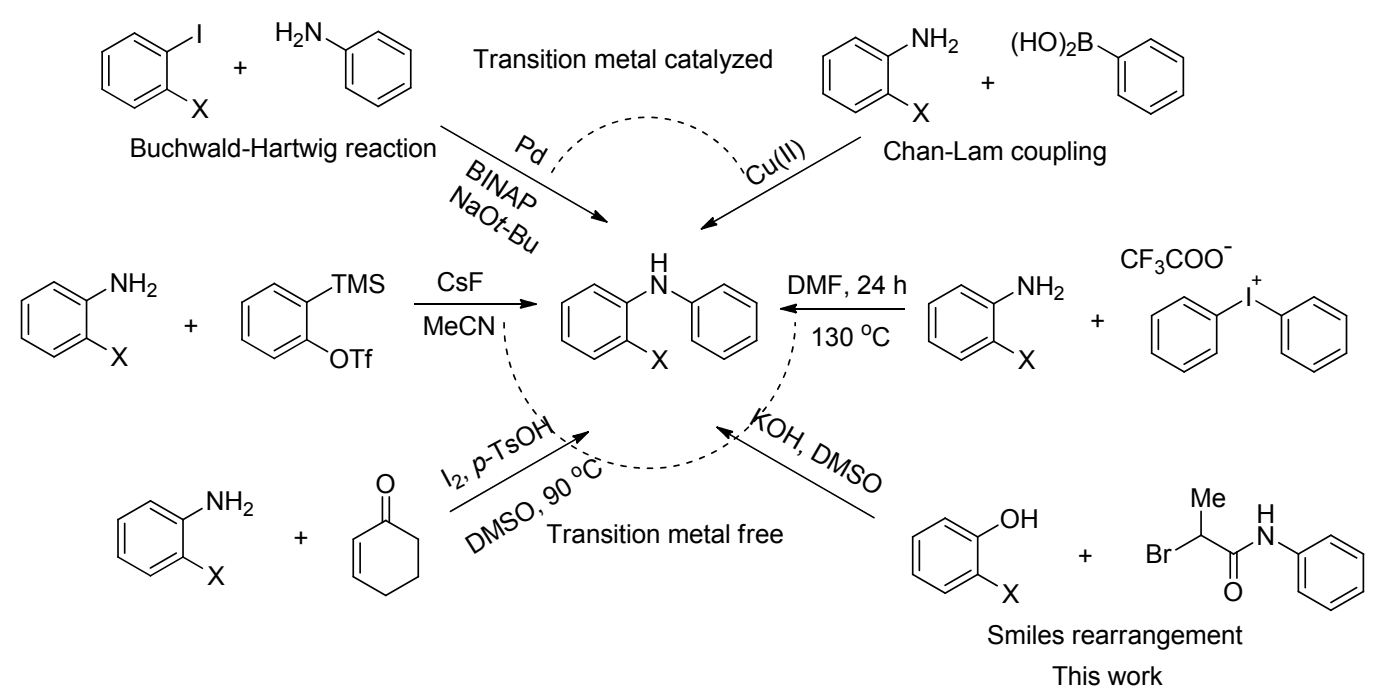

图式 1 之前的研究和本工作

Scheme 1 Previous studies and this work

\section{1 结果与讨论}

\section{1 反应条件的选择}

我们小组 ${ }^{[8]}$ 之前报道了一种非金属合成简单二芳胺 化合物的方法. 本文设想通过反应条件的优化从邻卤苯 酚出发合成邻卤二芳胺类化合物. 为此, 我们先在 $50{ }^{\circ} \mathrm{C}$ 条件下, 以邻碘苯酚(1a)和 2-溴- $N$-(4-甲氧苯基)丙 酰胺 (2a) 为起始原料合成了中间产物 2-(2-碘苯氧 基)- $N$-(4-甲氧苯基)丙酰胺(3a) (Eq. 1). 然后, 以 $\mathbf{3 a}$ 作为 模型化合物, 进行反应条件的篮选和优化, 实验结果如 表 1 所示. 以 $\mathrm{KOH}$ 为碱 ${ }^{[8]}$ 可获得目标产物 $\mathbf{4 a}$, 但收率只 有 26\%, 同时得到副产物 $\mathbf{4 a b}$, 收率为 33\% (表 1, Entry 1). 试用碱性较弱的 $\mathrm{Cs}_{2} \mathrm{CO}_{3}$ 或者 $\mathrm{K}_{2} \mathrm{CO}_{3}$, 转化率却急剧 下降(表 1, Entries 2,3). 但试用更强的碱 $\mathrm{KO}^{t} \mathrm{Bu}$ 可以提 高转化率, 但没有分离到 3a 且只以 $46 \%$ 的收率得到了

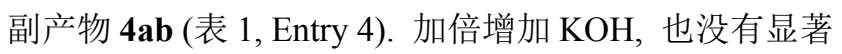
地提高产物的收率(表 1, Entry 5). 设想能否改变碱的加 入方式来提高收率, 我们在 $130{ }^{\circ} \mathrm{C}$ 下分两次等量加入 $\mathrm{KOH}$, 反应仍然不理想(表 1, Entry 6). 尝试微波加热也 没有得到良好的结果(表 1, Entry 7). 最后, 我们发现加 入 1.2 equiv. 的 $\mathrm{KOH}$ 后在 $130{ }^{\circ} \mathrm{C}$ 反应 $2 \mathrm{~h}$, 然后降低温度 至 $50{ }^{\circ} \mathrm{C}$ 后再加入等量的 $\mathrm{KOH}$, 升温至 $130{ }^{\circ} \mathrm{C}$ 反应 $2 \mathrm{~h}$.<smiles>COc1ccc(NC(=O)C(C)Oc2ccccc2I)cc1</smiles>

这种改进的方法获得了良好的结果，以 $66 \%$ 的收率得到 了目标产物 3a(表 1, Entry 8).

表 1 合成邻卤二芳胺 $4 \mathrm{a}$ 的反应条件优化 ${ }^{a}$

Table 1 Optimized condition for the synthesis of $o$-halodiarylamines 4a

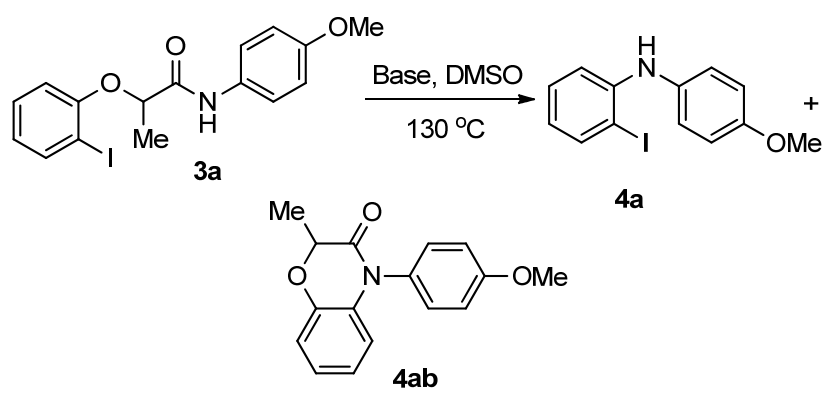

\begin{tabular}{cllcccc}
\hline \multirow{2}{*}{ Entry } & \multirow{2}{*}{ Base } & \multirow{2}{*}{ Base/equiv. } & \multirow{2}{*}{ Time/h } & \multirow{2}{*}{ Conv./\% } & \multicolumn{3}{c}{ Yield $^{b} \%$} \\
\cline { 5 - 7 } & & & & & 4a & 4ab \\
\hline 1 & $\mathrm{KOH}$ & 1.2 & 2 & 57 & 26 & 33 \\
2 & $\mathrm{Cs}_{2} \mathrm{CO}_{3}$ & 1.2 & 12 & 38 & 24 & 13 \\
3 & $\mathrm{~K}_{2} \mathrm{CO}_{3}$ & 1.2 & 12 & 0 & 0 & 0 \\
4 & $\mathrm{KO}{ }^{t} \mathrm{Bu}$ & 1.2 & 2 & $>99$ & 0 & 46 \\
5 & $\mathrm{KOH}$ & 2.4 & 4 & - & $30^{c}$ & - \\
$6^{d}$ & $\mathrm{KOH}$ & $1.2+1.2$ & $2+2$ & - & $33^{c}$ & - \\
$7^{e}$ & $\mathrm{KOH}$ & $1.2+1.2$ & $1+1$ & - & $28^{c}$ & - \\
$8^{f}$ & $\mathrm{KOH}$ & $1.2+1.2$ & $2+2$ & - & $66^{c}$ & - \\
\hline
\end{tabular}

${ }^{a}$ Reagents and conditions: $3 \mathbf{a}(0.2 \mathrm{mmol})$, base $(0.24 \mathrm{mmol})$ and DMSO $(0.6$ $\mathrm{mL}$ ) were heated from room temperature to $130{ }^{\circ} \mathrm{C} ;{ }^{b}$ Determined by ${ }^{1} \mathrm{H}$ NMR of the crude reaction mixture; ${ }^{c}$ Isolated yield; ${ }^{d}$ The base was added by two batches evenly each time at $130{ }^{\circ} \mathrm{C} ;{ }^{e}$ The reaction mixture was heated by microwave and the base was added by two batches evenly each time; ${ }^{f}$ The second dose base was added after the mixture was cooled to $50{ }^{\circ} \mathrm{C}$.

\section{2 底物拓展}

为了考察该合成方法的普遍适用性，在上述最佳反 应条件下进行了底物拓展研究(表 2). 首先, 我们考察了 2-碘苯酚与 2-澳- $N$-(芳基)丙酰胺(2)的反应. 当苯环的对 
位是供电子基(如甲氧基和甲基)时，反应能顺利进行， 以较好的产率得到目标产物 $4 \mathrm{a}$ 和 $4 \mathrm{c}$. 当苯环的对位是 吸电子基团(如氟、氯和溴)时, 产物 $4 \mathrm{~d} 、 4 \mathrm{e}$ 和 $4 \mathrm{f}$ 的产率 相对较低. 当苯环的邻位是甲基时, 没有得到目标产物 4g. 而苯环的间位为甲基时, 以 $53 \%$ 的产率得到目标产 物 4h. 然后, 我们考察了 2-溴取代苯酚与 2-溴- $N$-(芳基) 丙酰胺 2 的反应. 无论 2-溴- $N$-(芳基)丙酰胺带有供电子 基(如甲氧基和甲基), 还是带有吸电子基团(如氟和氯) 都可以和 4-甲基-2-溴苯酚反应，以中等产率得到产物
$4 j 、 4 k 、 4 l$ 和 $4 m$. 同时也考察了其它 4-取代-2-溴苯酚 参与的反应，分别得到了相应的产物 $4 \mathrm{n}$ 和 40 . 有趣的 是，1-溴-2-菜酚也可以与 2-溴- $N$-(甲氧苯基)丙酰胺 (2a) 反应，以 $38 \%$ 的产率得到了产物 $\mathbf{4 p}$.

\section{3 可能的反应机理}

基于上述实验结果，我们提出了可能的反应机理 (Scheme 2) ${ }^{[9]}$. 第一步是 3 在强碱作用下通过去质子化 生成酰胺负离子 $\mathbf{A}^{[10]}$. 负离子 $\mathbf{A}$ 进攻芳环形成五元环状

表 2 非金属方法合成邻卤二芳胺 $\mathbf{4}^{a}$

Table 2 Metal-free synthesis of $o$-halodiarylamines 4

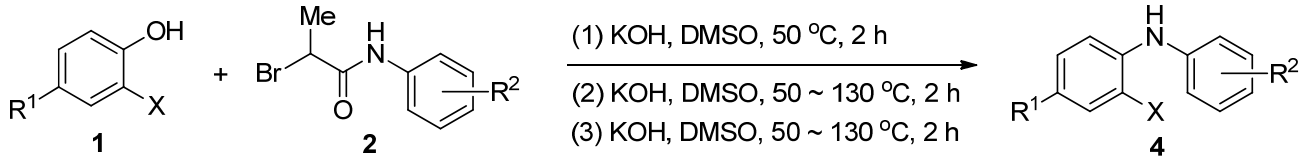<smiles>COc1ccc(Nc2ccccc2I)cc1</smiles>

4a $66 \%$<smiles>Ic1ccccc1Nc1ccccc1I</smiles>

4b $67 \%$<smiles>Cc1ccc(Nc2ccccc2I)cc1</smiles>

4c $81 \%$<smiles>Fc1ccc(Nc2ccccc2I)cc1</smiles>

4d $27 \%$<smiles>Clc1ccc(Nc2ccccc2I)cc1</smiles>

4e $32 \%$<smiles>Brc1ccc(Nc2ccccc2I)cc1</smiles>

4f $36 \%$<smiles>Cc1ccccc1Nc1ccccc1I</smiles>

$4 g$ N.R.<smiles>Cc1cccc(Nc2ccccc2I)c1</smiles>

4h $53 \%$<smiles>Brc1ccccc1Nc1ccccc1</smiles>

4i $75 \%$<smiles>COc1ccc(Nc2ccc(C)cc2Br)cc1</smiles><smiles>Cc1ccc(Nc2ccc(C)cc2Br)cc1</smiles>

4j $57 \%$<smiles>Cc1ccc(Nc2ccc(F)cc2Br)c(Br)c1</smiles>

4l $55 \%$

4m $50 \%$<smiles>COc1ccc(Nc2ccc(Cl)cc2Br)cc1</smiles>

4n $23 \%$<smiles>COc1ccc(Nc2ccc(C(C)(C)C)cc2Br)cc1</smiles>

4o $64 \%$<smiles>COc1ccc(Nc2cccc3ccccc23)cc1</smiles>

${ }^{a}$ Reagents and conditions: (1) $o$-Halophenols 1 (1. 0 mmol), 2-bromo- $N$-arylpropanamide 2 (1.2 mmol), $\mathrm{KOH}(1.2 \mathrm{mmol}), \mathrm{DMSO}(3 \mathrm{~mL}), 50{ }^{\circ} \mathrm{C}, 2 \mathrm{~h}$; (2) KOH (1.2 mmol), $130{ }^{\circ} \mathrm{C}, 2 \mathrm{~h}$; (3) $\mathrm{KOH}(1.2 \mathrm{mmol})$ was added after the mixture was cooled to $50{ }^{\circ} \mathrm{C}$, then the mixture was heated to $130{ }^{\circ} \mathrm{C}$ again and stirred for $2 \mathrm{~h}$; isolated yields were given.

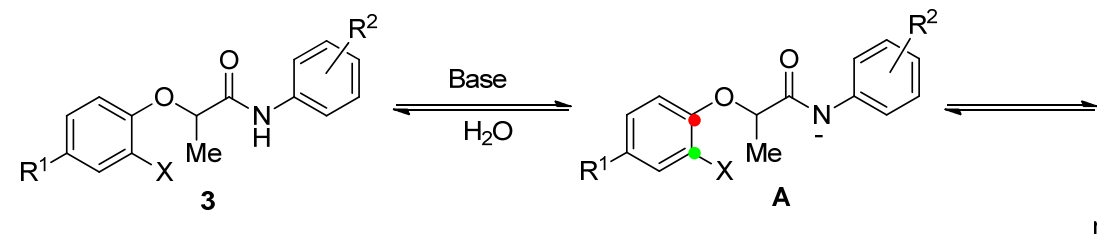<smiles>[R]c1ccc(N2C(=O)C(C)OC(C)(C)C2C)cc1</smiles>

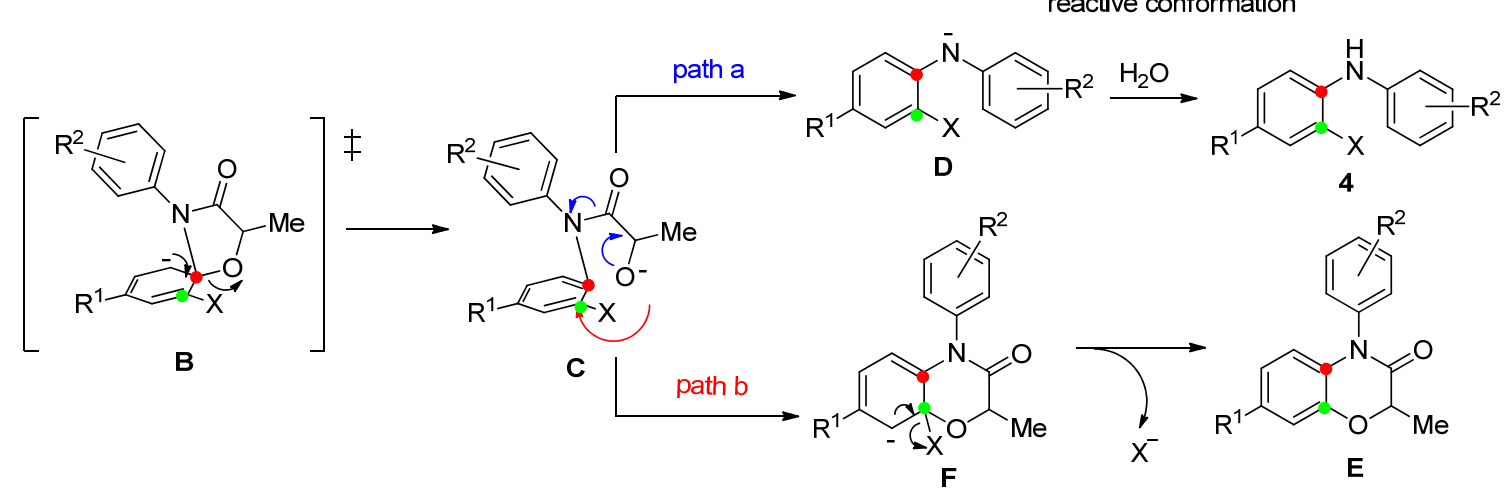

图式 2 可能的反应机理

Scheme 2 Possible reaction mechanism 
过渡态 $\mathbf{B}^{[11]}, \mathbf{B}$ 进而生成中间体 $\mathbf{C}$. 从 $\mathbf{3}$ 到关键中间体 $\mathbf{C}$ 的转化是一种典型的碱催化的 Smiles 重排反应. 从中间 体 $\mathbf{C}$ 通过途径 $\mathrm{a}$ 得到苯胺负离子 $\mathbf{D}$, 进一步生成最终产 物 4 , 这一步涉及到酰胺键的 $\mathrm{C}-\mathrm{N}$ 键断裂. 含氧负离子 $\mathbf{C}$ 也可通过途径 $b$, 进攻苯环得到中间体 $\mathbf{F}$, 然后 $\mathbf{F}$ 中的 卤素负离子离去最终生成副产物 $\mathbf{E}^{[12]}$.

\section{2 结论}

我们发展了一种 $\mathrm{KOH} / \mathrm{DMSO}$ 促进的由邻卤苯酚为 原料经 Smiles 重排反应合成邻卤二苯胺的方法. 这种绿 色、简便的合成方法在有机合成化学和其它方面将得到 很好的应用.

\section{3 实验部分}

\section{1 仪器与试剂}

WRR 熔点仪(上海申光仪器仪表有限公司, 温度未 经校正); Bruker Advance-400 FT 型核磁共振仪(德国 Bruker 公司, 溶剂为 $\mathrm{CDCl}_{3}$, TMS 为内标); GCT Premier 气相色谱高分辨飞行时间质谱联用仪(美国 WATERS 公 司); NICOLET iS10 傅里叶红外光谱仪(美国 Thermo Fisher Scientific 公司); 紫外灯检测(UV-8 三用紫外仪); 薄层色谱用硅胶(GF254, 青岛海洋化工厂产品). 其余 试剂均为国产化学纯或分析纯.

\section{2 实验方法}

\subsubsection{2-溴- $N$-(芳基)丙酰胺 $\mathbf{2}$ 的合成}

向 $100 \mathrm{~mL}$ 反应瓶中先后加入芳胺 $(20 \mathrm{mmol}) 、 2$-溴 丙酸 $(21 \mathrm{mmol})$ 和 $30 \mathrm{~mL}$ 二氯甲烷, 搅拌溶解, 加入 4 二 甲基吡啶 (DMAP, $0.2 \mathrm{mmol}$ ). 将反应混合物置于 $0{ }^{\circ} \mathrm{C}$ 冰水浴中, 分批加入二环已基碳二亚胺(DCC, 21 $\mathrm{mmol}$ ), 摚拌过夜. 反应液用硅胶抽滤后, 滤液旋干. 用 乙醇重结晶后, 得 2-溴- $N$-(芳基)丙酰胺 $\mathbf{2}$, 直接用于下 一步反应.

\subsection{2 邻卤二芳胺的合成}

向 $25 \mathrm{~mL}$ 反应瓶中加入邻卤苯酚 $1(1.0 \mathrm{mmol}) 、 2$ 溴- $N$-(芳基)丙酰胺 $2(1.2 \mathrm{mmol}) 、 \mathrm{KOH}(1.2 \mathrm{mmol})$ 和溶 剂 DMSO $(3 \mathrm{~mL})$, 在 $50{ }^{\circ} \mathrm{C}$ 下搅拌反应 $2 \mathrm{~h}$, 然后再加入 $\mathrm{KOH}(1.2 \mathrm{mmol})$, 加热到 $130{ }^{\circ} \mathrm{C}$ 后继续摚拌反应 $2 \mathrm{~h}$. 反应温度降至 $50{ }^{\circ} \mathrm{C}$, 再加入 $\mathrm{KOH}(1.2 \mathrm{mmol})$, 升温到 $130{ }^{\circ} \mathrm{C}$ 继续搅拌反应 $2 \mathrm{~h}$. 冷却, 用 $10 \mathrm{~mL}$ 的饱和氯化钠 溶液淬灭反应, 乙酸乙酯 $(10 \mathrm{~mL} \times 3)$ 萃取, 无水 $\mathrm{Na}_{2} \mathrm{SO}_{4}$ 干燥, 过滤, 将滤液旋蒸, 剩余物质用硅胶柱层析 (乙 酸乙酯/石油醚作展开剂梯度洗脱)分离得到邻卤二芳胺 4.

2-碘- $N$-(4- 甲氧苯基)苯胺 (4a)：黄色液体，产率
66\%. ${ }^{1} \mathrm{H}$ NMR (400 MHz, $\left.\mathrm{CDCl}_{3}\right) \delta: 7.71(\mathrm{dd}, J=7.9,1.4$ $\mathrm{Hz}, 1 \mathrm{H}), 7.17 \sim 7.06(\mathrm{~m}, 3 \mathrm{H}), 6.93 \sim 6.83(\mathrm{~m}, 3 \mathrm{H}), 6.52$ (td, $J=7.8,1.5 \mathrm{~Hz}, 1 \mathrm{H}), 5.78(\mathrm{~s}, 1 \mathrm{H}), 3.81(\mathrm{~s}, 3 \mathrm{H}) ;{ }^{13} \mathrm{C}$ NMR $\left(100 \mathrm{MHz}, \mathrm{CDCl}_{3}\right) \delta: 156.48,145.81,139.45$, $134.61,129.20,124.70,120.63,114.88,113.87,86.71$, 55.68; IR (KBr) v: 3375, 3060, 2998, 2952, 2832, 1583, $1511,1450,1398,1295,1245,1180,1106,1036,1007$, $817,744,612 \mathrm{~cm}^{-1}$; HRMS (EI-TOF) calcd for $\mathrm{C}_{13} \mathrm{H}_{12} \mathrm{NOI}$ $\left(\mathrm{M}^{+}\right)$324.9964, found 324.9969 .

2-碘- $N$ - 苯基苯胺 $(\mathbf{4 b})^{[13]}$ ：黄色油状液体，产率 67.3\%. ${ }^{1} \mathrm{H}$ NMR $\left(400 \mathrm{MHz}, \mathrm{CDCl}_{3}\right) \delta: 7.80 \sim 7.73(\mathrm{~m}$, $1 \mathrm{H}), 7.35 \sim 7.26(\mathrm{~m}, 2 \mathrm{H}), 7.22 \sim 7.08(\mathrm{~m}, 4 \mathrm{H}), 7.07 \sim 6.98$ (m, 1H), 6.61 (ddd, $J=8.0,5.4,3.4 \mathrm{~Hz}, 1 \mathrm{H}), 5.90(\mathrm{~s}, 1 \mathrm{H})$; ${ }^{13} \mathrm{C}$ NMR (100 MHz, $\left.\mathrm{CDCl}_{3}\right) \delta: 144.03,142.10,139.65$, 129.59, 129.16, 122.67, 122.04, 120.11, 115.95, 88.89; IR (KBr) $v$ : 3381, 3058, 1590, 1578, 1505, 1461, 1446, 1413, $1310,1284,1218,1175,1159,1077,1008,882,744,693$, $636,574 \mathrm{~cm}^{-1}$.

2-碘- $N$-(对甲苯基) 苯胺 $(\mathbf{4 c})^{[14]}$ : 棕色液体, 产率 81\%. ${ }^{1} \mathrm{H}$ NMR (400 MHz, $\mathrm{CDCl}_{3}$ ) $\delta: 7.74$ (dd, $J=7.9,1.4$ $\mathrm{Hz}, 1 \mathrm{H}), 7.20 \sim 7.01(\mathrm{~m}, 6 \mathrm{H}), 6.57(\mathrm{td}, J=7.9,1.6 \mathrm{~Hz}$, $1 \mathrm{H}), 5.85(\mathrm{~s}, 1 \mathrm{H}), 2.33(\mathrm{~s}, 3 \mathrm{H}) ;{ }^{13} \mathrm{C}$ NMR $(100 \mathrm{MHz}$, CDCl3) $\delta: 144.74,139.56,139.27,132.79,130.14,129.16$, 121.34, 121.29, 114.99, 87.94, 20.97; IR (KBr) v: 3381, 2918, 1587, 1515, 1450, 1396, 1309, 1219, 1109, 1008, $806,743 \mathrm{~cm}^{-1}$.

2 -碘- $N$-(4-氟苯基)苯胺 $(4 \mathbf{d})$ ：无色液体，产率 $27 \%$. ${ }^{1} \mathrm{H}$ NMR $\left(400 \mathrm{MHz}, \mathrm{CDCl}_{3}\right) \delta: 7.75(\mathrm{dd}, J=8.0,1.7 \mathrm{~Hz}$, $1 \mathrm{H}), 7.17$ (ddd, $J=8.7,7.2,1.6 \mathrm{~Hz}, 1 \mathrm{H}), 7.11$ (dd, $J=9.3$, $4.9 \mathrm{~Hz}, 3 \mathrm{H}), 7.07 \sim 6.94(\mathrm{~m}, 6 \mathrm{H}), 6.64 \sim 6.55(\mathrm{~m}, 1 \mathrm{H}) ;{ }^{13} \mathrm{C}$ NMR (100 MHz, $\left.\mathrm{CDCl}_{3}\right) \delta: 159.13(\mathrm{~d}, J=240.7 \mathrm{~Hz})$, $144.80,139.64,137.93,129.27,123.27\left(\mathrm{~d}, J_{\mathrm{C}-\mathrm{F}}=8.0 \mathrm{~Hz}\right)$, $121.64,116.31\left(\mathrm{~d}, J_{\mathrm{C}-\mathrm{F}}=22.4 \mathrm{~Hz}\right), 114.85,87.86$; IR (KBr) $v: 3381,3059,1604,1583,1506,1453,1395,1310,1212$, 1154, 1096, 1008, 820, 783, $744 \mathrm{~cm}^{-1}$. HRMS (EI-TOF) calcd for $\mathrm{C}_{12} \mathrm{H}_{9}$ NIF 312.9764, found 312.9764.

2 -碘- $N$-(4-氯苯基)苯胺 $(4 \mathbf{e})$ ：黄色油状液体，产率 $32 \% .{ }^{1} \mathrm{H}$ NMR (400 MHz, $\mathrm{CDCl}_{3}$ ) $\delta: 7.78$ (dd, $J=7.9,1.4$ $\mathrm{Hz}, 1 \mathrm{H}), 7.30 \sim 7.24$ (m, 5H), 7.21 (ddd, $J=8.5,7.2,1.4$ $\mathrm{Hz}, 1 \mathrm{H}), 7.14(\mathrm{dd}, J=8.2,1.6 \mathrm{~Hz}, 1 \mathrm{H}), 7.09 \sim 7.00(\mathrm{~m}$, $2 \mathrm{H}), 6.69 \sim 6.60(\mathrm{~m}, 1 \mathrm{H}), 5.86(\mathrm{~s}, 1 \mathrm{H}) ;{ }^{13} \mathrm{C}$ NMR $(100$ $\left.\mathrm{MHz}, \mathrm{CDCl}_{3}\right) \delta: 143.63,140.86,139.76,129.59,129.28$, $127.35,122.60,121.08,116.32,89.28$; IR (KBr) v: 3380, $1585,1493,1453,1310,1175,1091,1010,813,747 \mathrm{~cm}^{-1}$. HRMS (EI-TOF) calcd for $\mathrm{C}_{12} \mathrm{H}_{9} \mathrm{NClI} 328.9467$, found 
328.9468 .

2-碘- $N$-(4-溴苯基)苯胺 $(\mathbf{4 f})$ ：棕色液体，产率 $36 \%$. ${ }^{1} \mathrm{H}$ NMR (400 MHz, $\left.\mathrm{CDCl}_{3}\right) \delta: 7.78(\mathrm{dd}, J=7.9,1.3 \mathrm{~Hz}$, $1 \mathrm{H}), 7.44 \sim 7.35(\mathrm{~m}, 2 \mathrm{H}), 7.25 \sim 7.14(\mathrm{~m}, 2 \mathrm{H}), 7.03 \sim 6.95$ $(\mathrm{m}, 2 \mathrm{H}), 6.72 \sim 6.62(\mathrm{~m}, 1 \mathrm{H}), 5.85(\mathrm{~s}, 1 \mathrm{H}) ;{ }^{13} \mathrm{C}$ NMR $(100$ $\left.\mathrm{MHz}, \mathrm{CDCl}_{3}\right) \delta: 143.44,141.45,139.78,132.52,129.28$, $122.78,121.21,116.60,114.58,89.54$; IR (KBr) v: 2932, $1715,1580,1506,1464,1355,1294,1245,1178,1124$, 1032, 915, 835, $684 \mathrm{~cm}^{-1}$. HRMS (EI-TOF) calcd for $\mathrm{C}_{12} \mathrm{H}_{9} \mathrm{NBrI} 372.8962$, found 372.8963 .

2-碘- $N$-(间甲苯基)苯胺(4h)：黄色液体，产率 $53 \%$. ${ }^{1} \mathrm{H}$ NMR (400 MHz, $\left.\mathrm{CDCl}_{3}\right) \delta: 7.80 \sim 7.73(\mathrm{~m}, 1 \mathrm{H})$, $7.23 \sim 7.15(\mathrm{~m}, 3 \mathrm{H}), 6.94(\mathrm{~d}, J=6.5 \mathrm{~Hz}, 2 \mathrm{H}), 6.89 \sim 6.82$ $(\mathrm{m}, 1 \mathrm{H}), 6.65 \sim 6.56(\mathrm{~m}, 1 \mathrm{H}), 2.33(\mathrm{~s}, 3 \mathrm{H}) ;{ }^{13} \mathrm{C} \mathrm{NMR}(100$ $\left.\mathrm{MHz}, \mathrm{CDCl}_{3}\right) \delta: 143.98,141.89,139.49,139.42,129.27$, $129.02,123.41,121.77,120.68,117.01,115.88,88.68$, 21.49; IR (KBr) v: 3382, 3035, 2918, 2857, 1693, 1607, $1579,1510,1488,1462,1441,1406,1311,1249,1167$, 1113, 1089, 1043, 1008, 941, 743, 692, 668, $650 \mathrm{~cm}^{-1}$. HRMS (EI-TOF) calcd for $\mathrm{C}_{13} \mathrm{H}_{12} \mathrm{NI} 309.0015$, found 309.0012 .

2 -溴- $N$-苯基苯胺 $(4 \mathbf{i})^{[14]}$ : 黄色油状液体, 产率 $75 \%$. ${ }^{1} \mathrm{H}$ NMR (400 MHz, $\mathrm{CDCl}_{3}$ ) $\delta: 7.52(\mathrm{dd}, J=8.0,1.4 \mathrm{~Hz}$, $2 \mathrm{H}), 7.37 \sim 7.29(\mathrm{~m}, 4 \mathrm{H}), 7.25(\mathrm{dd}, J=6.8,1.3 \mathrm{~Hz}, 2 \mathrm{H})$, $7.20 \sim 7.11(\mathrm{~m}, 6 \mathrm{H}), 7.09 \sim 6.99(\mathrm{~m}, 2 \mathrm{H}), 6.73(\mathrm{ddd}, J=$ 8.0, 7.3, $1.6 \mathrm{~Hz}, 2 \mathrm{H}), 6.08$ (s, 1H); ${ }^{13} \mathrm{C}$ NMR (100 MHz, $\left.\mathrm{CDCl}_{3}\right) \delta: 141.72,133.11,129.59,128.23,122.83,121.03$, 120.39, 115.91, 112.30; IR (KBr) v: 3396, 3062, 3040, $1592,1505,1464,1450,1415,1313,1239,1219,1176$, $1158,1123,1078,1044,1021,884,743,694,666,637$, $575 \mathrm{~cm}^{-1}$.

2-溴- $N$-(4-甲氧基苯基)-4-甲基苯胺 $(\mathbf{4 j}$ ): 黄色液体, 产率 57\%. ${ }^{1} \mathrm{H}$ NMR (400 MHz, $\left.\mathrm{CDCl}_{3}\right) \delta: 7.32(\mathrm{~s}, 1 \mathrm{H})$, $7.25(\mathrm{~s}, 0 \mathrm{H}), 7.09$ (d, J=8.8 Hz, 2H), $6.95 \sim 6.84(\mathrm{~m}, 4 \mathrm{H})$, $5.79(\mathrm{~s}, 1 \mathrm{H}), 3.80(\mathrm{~s}, 3 \mathrm{H}), 2.24(\mathrm{~s}, 3 \mathrm{H}) ;{ }^{13} \mathrm{C}$ NMR $(100$ $\left.\mathrm{MHz}, \mathrm{CDCl}_{3}\right) \delta: 156.05,140.72,134.98,133.15,129.69$, $128.87,123.87,114.81,114.69,111.01,77.48,77.16$, 76.84, 55.69, 20.28; IR (KBr) v: 3392, 2999, 2954, 2925, $2833,1595,1513,1455,1441,1400,1315,1295,1244$, 1180, 1107, 1044, 1107, 1044, 1020, 838, 817, 770, 743, $612,551 \mathrm{~cm}^{-1}$. HRMS (EI-TOF) calcd for $\mathrm{C}_{14} \mathrm{H}_{14} \mathrm{NI}$ 323.0171, found 323.0168.

2-溴- $N$-(对甲苯基)4-甲基苯胺(4k): 黄色液体，产 率 53\%. ${ }^{1} \mathrm{H}$ NMR (400 MHz, $\left.\mathrm{CDCl}_{3}\right) \delta: 7.34(\mathrm{~d}, J=1.5$ $\mathrm{Hz}, 1 \mathrm{H}), 7.24$ (s, 1H), $7.14 \sim 7.07(\mathrm{~m}, 2 \mathrm{H}), 7.01(\mathrm{~d}, J=8.4$
$\mathrm{Hz}, 2 \mathrm{H}), 6.94$ (dd, $J=8.3,1.6 \mathrm{~Hz}, 1 \mathrm{H}), 5.87$ (s, 1H), 2.31 $(\mathrm{s}, 3 \mathrm{H}), 2.25(\mathrm{~s}, 3 \mathrm{H}) ;{ }^{13} \mathrm{C} \mathrm{NMR}\left(100 \mathrm{MHz}, \mathrm{CDCl}_{3}\right) \delta$ : $139.64,139.54,133.26,132.08,130.52,130.06,128.84$, 120.43, 115.96, 112.12, 77.48, 77.16, 76.84, 20.90, 20.35; IR (KBr) v: 3399, 3024, 2918, 2852, 1605, 1515, 1387, $1312,1277,1225,1117,1102,1037,869,808,697,669$ $\mathrm{cm}^{-1}$. HRMS (EI-TOF) calcd for $\mathrm{C}_{14} \mathrm{H}_{14} \mathrm{NBr} 275.0310$, found 275.0310 .

2-溴- $N$-(4-氟苯基)-4-甲基苯胺(41): 黄色液体, 产率 $55 \%$. ${ }^{1} \mathrm{H}$ NMR (400 MHz, $\left.\mathrm{CDCl}_{3}\right) \delta: 7.37 \sim 7.32$ (m, 1H), $7.10 \sim 7.03(\mathrm{~m}, 2 \mathrm{H}), 7.03 \sim 6.92(\mathrm{~m}, 4 \mathrm{H}), 5.81(\mathrm{~s}, 1 \mathrm{H})$, $2.25(\mathrm{~s}, 3 \mathrm{H}) ;{ }^{13} \mathrm{C}$ NMR (100 MHz, $\left.\mathrm{CDCl}_{3}\right) \delta: 158.73(\mathrm{~d}$, $J=239.9), 139.56,138.27\left(\mathrm{~d}, J_{\mathrm{C}-\mathrm{F}}=26.0\right), 133.34,130.88$, $128.93,122.26\left(\mathrm{~d}, J_{\mathrm{C}-\mathrm{F}}=39.0\right), 116.28,115.93\left(\mathrm{~d}, J_{\mathrm{C}-\mathrm{F}}=\right.$ 25.5), 112.12, 77.48, 77.16, 76.84, 20.35; IR (KBr) v: 3401, 3035, 2920, 2855, 1608, 1514, 1505, 1386, 1314, $1277,1214,1155,1096,1038,1012,996,870,834,815$, 699, 670, $592 \mathrm{~cm}^{-1}$. HRMS (EI-TOF) calcd for $\mathrm{C}_{13} \mathrm{H}_{11} \mathrm{NFBr} 279.0059$, found 279.0060.

2-溴- $N$-(4-氯苯基)-4-甲基苯胺 $(4 \mathrm{~m})$ : 白色固体, 产 率 50\%. m.p. 63.7 $64.6{ }^{\circ} \mathrm{C} ;{ }^{1} \mathrm{H} \mathrm{NMR}(400 \mathrm{MHz}$, $\left.\mathrm{CDCl}_{3}\right) \delta: 7.40 \sim 7.35(\mathrm{~m}, 1 \mathrm{H}), 7.26 \sim 7.21(\mathrm{~m}, 2 \mathrm{H}), 7.12$ $(\mathrm{d}, J=8.3 \mathrm{~Hz}, 1 \mathrm{H}), 7.04 \sim 6.97(\mathrm{~m}, 3 \mathrm{H}), 2.28(\mathrm{~s}, 3 \mathrm{H}) ;{ }^{13} \mathrm{C}$ NMR (100 MHz, $\left.\mathrm{CDCl}_{3}\right) \delta: 141.26,138.32,133.50$, $132.01,129.50,128.96,126.66,120.22,117.37,113.39$, 20.44; IR (KBr) v: 3391, 3053, 3020, 2920, 1891, 1605, $1590,1507,1489,1428,1387,1308,1274,1223,1148$, 1084, 1035, 1013, 857, 825, 810, 717, 699, $545 \mathrm{~cm}^{-1}$. HRMS (EI-TOF) calcd for $\mathrm{C}_{13} \mathrm{H}_{11} \mathrm{NClBr} 294.9763$, found 294.9767.

2-溴- $N$-(4-甲氧苯基)-4-氯苯胺(4n): 黄色固体, 产 率 23\%. m.p. 83.4 85.1 ${ }^{\circ} \mathrm{C} ;{ }^{1} \mathrm{H}$ NMR (400 MHz, $\mathrm{CDCl}_{3}$ ) $\delta: 7.39 \sim 7.34(\mathrm{~m}, 1 \mathrm{H}), 7.27 \sim 7.18(\mathrm{~m}, 2 \mathrm{H}), 7.12(\mathrm{~d}, J=$ $8.3 \mathrm{~Hz}, 1 \mathrm{H}), 7.04 \sim 6.95(\mathrm{~m}, 3 \mathrm{H}), 2.27(\mathrm{~s}, 3 \mathrm{H}) ;{ }^{13} \mathrm{C} \mathrm{NMR}$ $\left(100 \mathrm{MHz}, \mathrm{CDCl}_{3}\right) \delta: 141.25,138.32,133.49,131.99$, 129.48, 128.95, 126.65, 120.20, 117.37, 113.40, 20.43; IR (KBr) v: 3394, 2965, 2932, 2836, 1588, 1561, 1510, 1494, $1486,1463,1442,1414,1389,1316,1290,1266,1257$, 1239, 1217, 1166, 1102, 1030, 862, 820, 806, 773, 697, $669,584 \mathrm{~cm}^{-1}$. HRMS (EI-TOF) calcd for $\mathrm{C}_{13} \mathrm{H}_{11} \mathrm{NClBr}$ 310.9713 , found 310.9709 .

2-溴- $N$-(4-甲氧苯基)-4-叔丁基苯胺(40): 黄色液体, 产率 64\%. ${ }^{1} \mathrm{H}$ NMR (400 $\left.\mathrm{MHz}, \mathrm{CDCl}_{3}\right) \delta: 7.49$ (d, $J=2.2$ $\mathrm{Hz}, 1 \mathrm{H}), 7.17 \sim 7.06(\mathrm{~m}, 3 \mathrm{H}), 6.95 \sim 6.84(\mathrm{~m}, 3 \mathrm{H}), 3.81(\mathrm{~s}$, $3 \mathrm{H}), 1.27(\mathrm{~s}, 9 \mathrm{H}) ;{ }^{13} \mathrm{C} \mathrm{NMR}\left(100 \mathrm{MHz}, \mathrm{CDCl}_{3}\right) \delta: 156.14$, 
$143.29,140.71,134.83,129.78,125.24,124.09,114.82$, $114.22,110.88,77.48,77.16,76.84,55.70,34.22,31.52$; IR (KBr) v: 3396, 3036, 2961, 2904, 2867, 2833, 1606, $1511,1463,1441,1391,1362,1317,1296,1280,1247$, 1180, 1115, 1105, 1037, 878, 842, 817, 772, 713, 664, 602 $\mathrm{cm}^{-1}$. HRMS (EI-TOF) $\mathrm{C}_{17} \mathrm{H}_{20} \mathrm{NOBr} 333.0728$, found 333.0723 .

1-溴- $N$-(4-甲氧苯基)菜-2-胺(4p): 棕色液体, 产率 $38 \%$. ${ }^{1} \mathrm{H}$ NMR (400 MHz, $\left.\mathrm{CDCl}_{3}\right) \delta: 8.08(\mathrm{~d}, J=8.5 \mathrm{~Hz}$, $1 \mathrm{H}), 7.69$ (d, $J=8.0 \mathrm{~Hz}, 1 \mathrm{H}), 7.61$ (d, $J=8.9 \mathrm{~Hz}, 1 \mathrm{H}), 7.52$ (ddd, $J=8.3,6.9,1.2 \mathrm{~Hz}, 1 \mathrm{H}), 7.33 \sim 7.27(\mathrm{~m}, 1 \mathrm{H}), 7.23$ $(\mathrm{d}, J=8.9 \mathrm{~Hz}, 1 \mathrm{H}), 7.19 \sim 7.14(\mathrm{~m}, 2 \mathrm{H}), 6.96 \sim 6.88(\mathrm{~m}$, $2 \mathrm{H}), 6.36(\mathrm{~s}, 1 \mathrm{H}), 3.83(\mathrm{~s}, 3 \mathrm{H}) ;{ }^{13} \mathrm{C} \mathrm{NMR}(100 \mathrm{MHz}$, $\left.\mathrm{CDCl}_{3}\right) \delta: 156.68,141.25,134.43,133.27,129.13,128.40$, $128.26,127.84,125.36,124.88,123.27,116.44,114.92$, 106.09, 77.48, 77.36, 77.16, 76.84, 55.71; IR (KBr) v: 3390, 3053, 3001, 2954, 2928, 2833, 1622, 1603, 1558, $1505,1463,1428,1409,1352,1282,1243,1180,1150$, 1106, 1035, 981, 938, 811, 761, 745, 661, $602 \mathrm{~cm}^{-1}$. HRMS (EI-TOF) calcd for $\mathrm{C}_{17} \mathrm{H}_{14} \mathrm{NOBr} 327.0259$, found 327.0261 .

2-甲基-4-(4-甲氧基苯基)苯并吗啉-3-酮(4ab) ${ }^{[15]}$ : 黄 色固体, m.p. 143.8 144.4 ${ }^{\circ} \mathrm{C}$; ${ }^{1} \mathrm{H}$ NMR (400 MHz, $\left.\mathrm{CDCl}_{3}\right) \delta: 7.18(\mathrm{~d}, J=8.8 \mathrm{~Hz}, 2 \mathrm{H}), 7.07 \sim 6.94(\mathrm{~m}, 4 \mathrm{H})$, $6.90 \sim 6.81(\mathrm{~m}, 1 \mathrm{H}), 6.44(\mathrm{dd}, J=8.1,1.3 \mathrm{~Hz}, 1 \mathrm{H}), 4.80$ (q, $J=6.8 \mathrm{~Hz}, 1 \mathrm{H}), 3.86(\mathrm{~s}, 3 \mathrm{H}), 1.64(\mathrm{~d}, J=6.8 \mathrm{~Hz}, 4 \mathrm{H}) ;{ }^{13} \mathrm{C}$ NMR (100 MHz, $\left.\mathrm{CDCl}_{3}\right) \delta: 167.04,159.65,144.24$, $131.24,129.89,128.87,124.03,122.52,117.36,116.81$, 115.33, 55.67, 16.52; IR (KBr) v: 3434, 2961, 2937, 2840, $1687,1609,1591,1512,1497,1462,1445,1371,1327$, $1297,1270,1253,1169,1130,1108,1049,1029,847,815$, 751, 684, 589, $576 \mathrm{~cm}^{-1}$. HRMS (EI-TOF) calcd for $\mathrm{C}_{16} \mathrm{H}_{15} \mathrm{NO}_{3} 269.1052$, found 269.1079.

\section{Referenes}

[1] (a) Yoo, E. J.; Chang, S. Org. Lett. 2008, 10, 1163.

(b) Xing, Y.; Sheng, G.; Wang, J.; Lu, P.; Wang, Y. Org. Lett. 2014, 16, 1244.

(c) Senadi, G. C.; Hu, W. P.; Boominathan, S. S. K.; Wang, J. J. Chem. Eur. J. 2015, 21, 998.

(d) Park, J. H.; Kim, E.; Chung, Y. K. Org. Lett. 2008, 10, 4719.

(e) Parisien, M.; Valette, D.; Fagnou, K. J. Org. Chem. 2005, 70, 7578 .

(f) Numata, M.; Yasuda, T.; Adachi, C. Chem. Commun. 2015, 51, 9443.

(g) Liu, Z.; Larock, R. C. Tetrahedron 2007, 63, 347.

(h) Li, X.; Chianese, A. R.; Vogel, T.; Crabtree, R. H.; Yang, Y.;
Zhang, X.; Zeng, W.; Huang, H.; Liang, Y. Org. Lett. 2005, 7, 5437. (i) Chen, Y.; Cho, C. H.; Shi, F.; Larock, R. C. J. Org. Chem. 2009, 74, 6802 .

(j) Andrew, T. L.; Swager, T. M. J. Org. Chem. 2011, 76, 2976.

(k) Fang, X.; Fang, L.; Gou, S. Chin. J. Org. Chem. 2012, 32, 1217 (in Chinese).

(房旭彬, 房雷, 苟少华, 有机化学, 2012, 32, 1217.)

(1) Xu, J.; Wei, Z.; Li, J. Chin. J. Org. Chem. 2012, 32, 1208 (in Chinese).

(徐娟, 魏真, 李加荣, 有机化学, 2012, 32, 1208.)

[2] (a) Guram, A. S.; Rennels, R. A.; Buchwald, S. L. Angew. Chem., Int. Ed. 1995, 34, 1348.

(b) Hartwig, J. F.; Kawatsura, M. S.; Hauck, I.; Shaughnessy, K. H.; Alcazar-Roman, L. M. J. Org. Chem. 1999, 64, 5575.

(c) Kuwano, R.; Utsunomiya, M.; Hartwig, J. F. J. Org. Chem. 2002, 67, 6479 .

(d) Gajare, A. S.; Toyota, K.; Yoshifuji, M.; Ozawa, F. J. Org. Chem. 2004, 69, 6504.

(e) Monguchi, Y.; Kitamoto, K.; Ikawa, T.; Maegawa, T.; Sajiki, H. Adv. Synth. Catal. 2008, 350, 2767.

(f) Roiban, G. D.; Mehler, G.; Reetz, M. T. Eur. J. Org. Chem. 2014, 2014, 2070.

(g) Topchiy, M. A.; Dzhevakov, P. B.; Rubina, M. S.; Morozov, O. S.; Asachenko, A. F.; Nechaev, M. S. Eur. J. Org. Chem. 2016, 1908.

[3] (a) Rivera-Utrilla, J.; Bautista-Toledo, I.; Feffo-Garcia, M. A.; Moreno-Castilla, C. Carbon 2003, 41, 323.

(b) Lam, P. Y. S.; Clark, C. G.; Saubern, S.; Adams, J.; Winters, M. P.; Chan, D. M. T.; Combs, A. Tetrahedron Lett. 1998, 39, 2941.

(c) Antilla, J. C.; Buchwald, S. L. Org. Lett. 2001, 3, 2077.

(d) Lan, J. B.; Zhang, G. L.; Yu, X. Q.; You, J. S.; Chen, L.; Yan, M.; Xie, R. G. Synlett 2004, 1095.

(e) Gogoi, A.; Sarmah, G.; Dewan, A.; Bora, U. Tetrahedron Lett. 2014, 55, 31 .

(f) Keesara, S. Tetrahedron Lett. 2015, 56, 6685.

(g) Yoo, W. J.; Tsukamoto, T.; Kobayashi, S. Angew. Chem., Int. Ed. 2015, 54, 6587.

(h) Roy, S.; Sarma, M. J.; Kashyap, B.; Phukan, P. Chem. Commun. 2016, 52, 1170.

[4] (a) Chan, D. M. T.; Monaco, K. L.; Wang, R. P.; Winters, M. P. Tetrahedron Lett. 1998, 39, 2933.

(b) Garrett, C. E.; Prasad, K. Adv. Synth. Catal. 2004, 346, 889.

(c) Lu, Q.; Yi, H.; Lei, A. Acta Chim. Sinica 2015, 73, 1245 (in Chinese).

(陆庆全，易红，雷爱文，化学学报, 2015, 73, 1245.)

[5] Liu, Z. J.; Larock, R. C. Org. Lett. 2003, 5, 4673.

[6] Carroll, M. A.; Wood, R. A. Tetrahedron 2007, 63, 11349.

[7] Barros, M. T.; Dey, S. S.; Maycock, C. D.; Rodrigues, P. Chem. Commun. 2012, 48, 10901.

[8] Yu, J. Z.; Wang, Y. T.; Zhang, P.; Wu, Z. J. Synlett 2013, 24, 1448.

[9] Yu, J. Z.; Zhang, P. Z.; Wu, J.; Shang, Z. C. Tetrahedron Lett. 2013, 54, 3167.

[10] Breugst, M.; Tokuyasu, T.; Mayr, H. J. Org. Chem. 2010, 75, 5250.

[11] Newman, M. S. Acc. Chem. Res. 1972, 5, 354.

[12] Zuo, H.; Meng, L. J.; Ghate, M.; Hwang, K. H.; Cho, Y. K.; Chandrasekhar, S.; Reddy, C. R.; Shin, D. S. Tetrahedron Lett. 2008, 49 , 3827.

[13] Liu, Z.; Larock, R. C. Org. Lett. 2003, 5, 4673.

[14] Barros, M. T.; Dey, S. S.; Maycock, C. D.; Rodrigues, P. Chem. Commun. 2012, 48, 10901.

[15] Feng, E.; Huang, H.; Zhou, Y.; Ye, D.; Jiang, H.; Liu, H. J. Org. Chem. 2009, 74, 2846. 